\title{
Introduction to the special section on managing system change in aviation: What makes for successful change?
}

\author{
Siobhán Corrigan • Nick McDonald
}

Published online: 1 October 2014

(c) Springer-Verlag London 2014

Within the aviation industry, the need for sustainable change is becoming more and more critical due to increasing commercial pressure, upcoming regulations and accelerating cycles of new technologies. But, whilst there is wide acceptance that change is necessary, the evidence is scant both in terms of empirical academic work and industrial-based case studies. Addressing the very real challenges industry has in implementing sustainable change was the key focus of the MAnaging System Change in Aviation (MASCA) project. MASCA was a three-year FP7 collaborative project funded by the European Union that finished in December 2013. The project was driven by the following four industry change initiatives:

- Case Study 1: Implementing Airport Collaborative Decision-Making (A-CDM).

- Case Study 2: The development and implementation of a comprehensive Safety Management System (SMS) in a major airline.

- Case Study 3: The development and implementation of a holistic performance management approach in a small regional airport.

- Case Study 4: The assessment of risk in advance of major change in a regional airline.

This special section puts forward a new approach to managing system change that has been tested and validated in collaborative industrial research and practice by means of the four case studies. The overall approach developed within the MASCA project provided a way of understanding some of the complexities of change processes and

S. Corrigan $(\bowtie) \cdot$ N. McDonald

Centre for Innovative Human Systems (CIHS), School of Psychology, Trinity College Dublin, Dublin, Ireland e-mail: siobhan.corrigan@tcd.ie to support better design and evaluation of each of the change initiatives. MASCA concluded with the following succinct, high-level definition of what is required to change an organisation:

At its most general, the capability of a resilient organisation has to encompass the following three characteristics:

To be able to mobilise its resources (especially its knowledge and the information that supports this) to anticipate future challenges, and to respond and adapt to such challenges (whether they are fully foreseen or not). In order to do this, it has to understand itself in two ways:

- how the system functions (how the interaction of human, social and technical aspects makes it possible to deliver value in the short, medium and long term); and

- what it is doing (how data from this activity are converted to knowledge about how the system is performing).

To have sufficient consensus to participate in and support the effective leadership of change.

This definition applies (all other things being equal) to a set of organisations functioning as an operational systemof-systems - as in aviation.

In order to support the development of this capability, MASCA has developed (or incorporated) an integrated set of services, methods and tools, which are summarised in Fig. 1, below. These are Managing information, Analysing, Serious gaming, Capability building and Achieving value (MASCA).

Operational and management processes are the core of the MASCA Change Management System; these should deliver value in real time, in a way that is both sustainable 
Fig. 1 Components of MASCA Change Management System

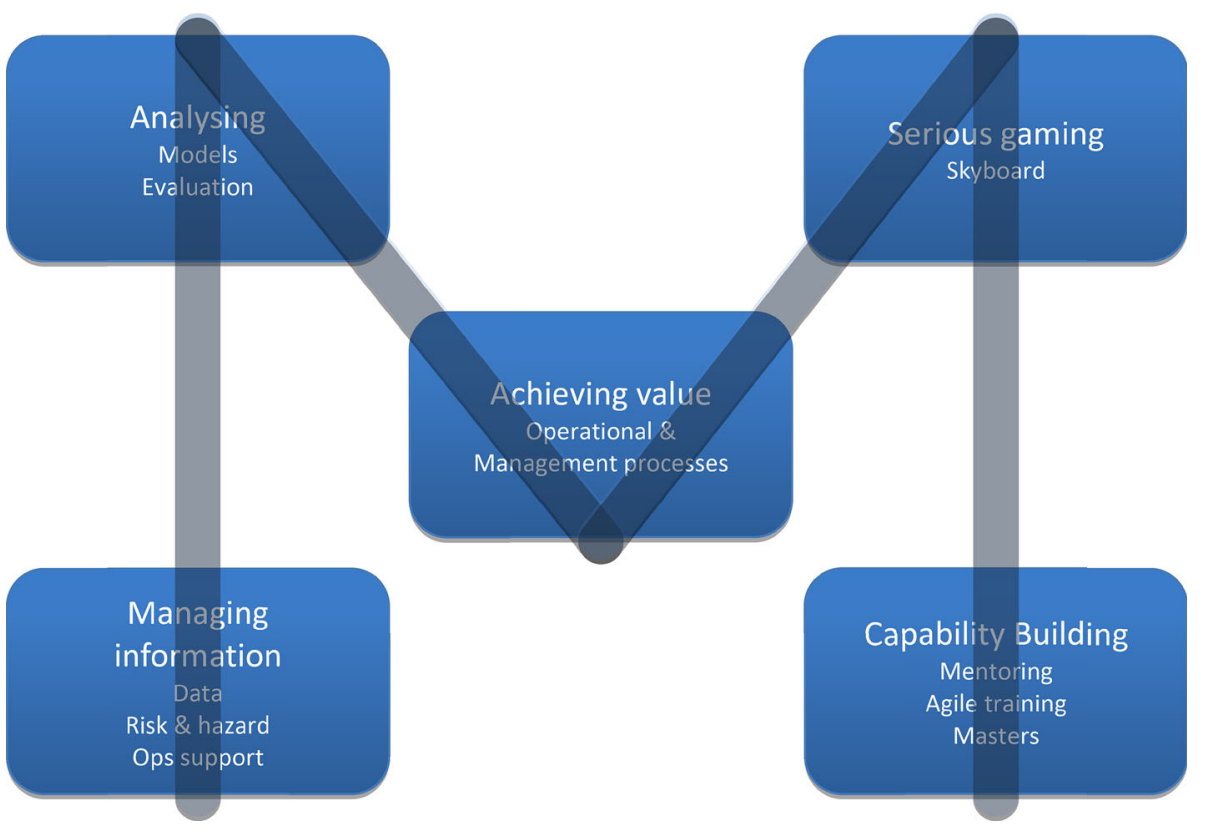

and capable of change (Achieving Value). Knowledge of these processes (the 'know-how' of how things really work) is captured in process maps, analyses and evaluations (Analysing). Data generated by process activity are gathered, integrated and assessed (Managing information). Capability building is supported by mentoring through external expertise; training is offered tailored to the needs of the initiative and the phase it is in; high-level competence in managing change is provided by a Masters program. Serious gaming provides participants with the opportunity to explore collaborative ways of working and to experiment with different roles and scenarios.

A core theme throughout the project has been the development of a theory or model of change that provides both a robust basis for supporting change initiatives and a coherent framework for evaluating their status and progress. The core proposition of the MASCA theoretical framework is that understanding the functionality of a sociotechnical system is the key to managing it more effectively. It focuses on three interlocking and interdependent levels of analysis: (1) process functionality (operational and management processes); (2) social cohesion (team and trust); and (3) collective knowledge and information cycles. McDonald in his paper provides a comprehensive description of the theory and a framework for the evaluation of the change initiatives that were undertaken as part of the MASCA project, summarises and consolidates the findings from the project case studies in terms of this overall framework.

This is followed by four papers focusing on each of the case studies identified above. Corrigan et al.'s paper provides a comprehensive and systematic evaluation into one airport's approach to preparing the ground work for full implementation of A-CDM. One of the key challenges centred around aligning goals of individual stakeholders (e.g. Air Traffic Control, Airport Authority, Airlines and Ground Handling) in order to facilitate the required level of collaboration to ensure more timely decision-making. Therefore, the evaluation highlighted the sections of social relations and social cohesion (particularly for multi-agency stakeholders), the right level of information and knowledge processes (that will engage people and make future systems work) and the capability of the new processes (both operational and management) to deliver the key strategic performance targets of A-CDM at the local airport levels and to the wider European Air-Space.

Ulfvengren and Corrigan's paper puts forward a conceptual framework for an integrated Safe-Lean programme and an evaluation of one airline's approach to developing and implementing a SMS. A comprehensive set of Safety Performance Indicators (SPI's) was initiated as a way of identifying and driving improvements. This involved challenging demands to develop a common safety and risk framework across different departments, to develop common performance management approaches between safety and other goals and to link safety with lean change initiatives. Leva et al.'s paper reports on the challenges facing a small regional airport in implementing a SMS. In this case study, a holistic performance management approach was developed supported primarily by the development of a web-based tool that can support, monitor and analyse dayto-day performance activities and anomalies. A database has also been designed to support the new SMS, which is still in process of development. Subtasks pertaining to each 
nonconformity are recorded in a dedicated Risk Register and analysed during scheduled meetings attended by staff and management in order to identify appropriate corrective actions. This is part of a major strategy to create a more business-oriented framework, which is critical to the organisation's survival.

The assessment of risk in advance of major change in a regional airline is the focus of the paper by Cacciabue et al. This case also focused on supporting the implementation of an SMS and illustrated a flexible reactive response to change imposed from outside by adapting conventional risk assessment methodology to adjust the company's response to the impact of that change. The final paper in this special section presents the overall learning process that evolved during the MASCA project, specifically focusing on one of the key elements of the overall learning approach, the development of a Serious Game (SKYBOARD), and the role the game played in supporting the implementation of A-CDM in a major European airport. The underlying principles of the learning process were based on ongoing and collaborative learning in the workplace, with each phase of learning involving preparation and guidance, collaborative learning, consolidation of that learning and practically focused next steps that can be deployed to support overall change management.

Overall, these cases represent work in progress, both in terms of practice and theory: the case studies continue as they are not bounded by the project, and the theory has to encompass this longitudinal dimension if it is ultimately to understand what makes for change that is relatively successful or otherwise. Thus, what is presented here is a cross-sectional snapshot of the different change initiatives at the current stage. Future work will report on their development and outcomes. What is presented in this special section is a proposition that understanding the internal dynamics of a sociotechnical system is one key to understanding how that system can be transformed and how that transformation can be supported. This in turn sets the agenda for leadership, the exercise of power and the role of culture. Thus, this work seeks to set a baseline upon which to build an empirically grounded theory that can provide practical support for more effective change management. 